\title{
On a generalized diffusion equation arising in petroleum engineering
}

\author{
Suliman Al-Homidan', Ryad A Ghanam² and Nasser-eddine Tatar ${ }^{\text {* }}$
}

${ }^{*}$ Correspondence:

tatarn@kfupm.edu.sa

${ }^{1}$ Department of Mathematics and

Statistics, King Fahd University of

Petroleum and Minerals, Dhahran,

31261, Saudi Arabia

Full list of author information is

available at the end of the article

\begin{abstract}
In this work we investigate a model which describes diffusion in petroleum engineering. The original model, which already generalizes the standard one (usual diffusion equation) to a non-local model taking into account the memory effects, is here further extended to cope with many other different possible situations. Namely, we consider the Hilfer fractional derivative which by its nature interpolates the Riemann-Liouville fractional derivative and the Caputo fractional derivative (the one that has been studied previously). At the same time, this kind of derivative provides us with a whole range of other types of fractional derivatives. We treat both the Neumann boundary conditions case and the Dirichlet boundary conditions case and find explicit solutions. In addition to that, we also discuss the case of an infinite reservoir.
\end{abstract}

\section{Introduction}

In order to modernize the public water service of the town of Dijon, France, Henry Darcy made several experiments and wrote an interesting document which soon formed the basis of the theory of fluid conduction. His work was about the download flow of water through filter sands. He established a (diffusion) equation, well known nowadays as 'Darcy's law', which plays the same role as Fourier's law in heat conduction theory and Ohm's law in the electricity conduction theory (see $[1,2])$. Of course, this law has its limitations. It is restricted to the situations where the flow through the pores can be modeled as Stokes flows. In particular, for large values of the Reynolds number (or at high values of flow rates) Darcy's law is not valid anymore. It losses its accuracy. Apart from that, Darcy's law has proved its usefulness in many applications such as the recovery of fuel from underground oil reservoirs. It defines the rock permeability (which controls the directional movement of the flow rate).

Many researchers have shown interest in this law and have worked extensively on extending and generalizing it to more complicated situations and in different contexts. For instance, they observed that this flow is 'linear': Darcy himself assumed that the flow is weak and as such the pressure drop is linearly related to the flow discharge rate. This motivated them to extend this law to the 'nonlinear' case. They also considered the case when the inertial forces (and/or the deformation of the solid) are not negligible as compared with those arising from viscosity. In particular, they generalized the law from Stokes to 'non-Stokes' flows in porous media. In fact, it has been found that more general physical formulations may be obtained by including new parameters and forces in the equation

○2013 Al-Homidan et al.; licensee Springer. This is an Open Access article distributed under the terms of the Creative Commons Attribution License (http://creativecommons.org/licenses/by/2.0), which permits unrestricted use, distribution, and reproduction in any medium, provided the original work is properly cited. 
itself. Moreover, we may mention here the transition from a single phase to multiphase and from fixed to growing porous media.

In [3] Caputo modified the standard diffusion equation

$$
\frac{\partial p}{\partial t}=k \frac{\partial^{2} p}{\partial x^{2}}
$$

by introducing a fractional derivative (in the sense of Caputo) in Darcy's law [1] to take into account the memory effects that may prevail in the fluid. The equation became

$$
\frac{\partial p}{\partial t}=k \frac{\partial^{2}}{\partial x^{2}}\left({ }^{c} D_{0^{+}}^{\alpha} p\right), \quad 0<\alpha<1
$$

where ${ }^{c} D_{0^{+}}^{\alpha}$ denotes the Caputo fractional derivative defined by

$$
\left({ }^{C} D_{0^{+}}^{\alpha} f\right)(t)=\frac{1}{\Gamma(1-\alpha)} \int_{0}^{t}(t-s)^{-\alpha} f^{\prime}(s) d s, \quad t>0,0<\alpha<1 .
$$

Similar equations have been derived in other situations like the one in [4] which describes anomalous subdiffusion particles (and $p$ is the probability density function) and in [5] when studying the Stokes first problem (see also [6]). A natural question which arises is: what about the commonly used Riemann-Liouville fractional derivative,

$$
\left({ }^{R L} D_{0^{+}}^{\alpha} f\right)(t)=\frac{1}{\Gamma(1-\alpha)} \frac{d}{d t} \int_{0}^{t}(t-s)^{-\alpha} f(s) d s, \quad t>0,0<\alpha<1,
$$

or other types of fractional derivatives?

It is well known by now that the Caputo fractional derivative is often preferred over the Riemann-Liouville fractional derivative at least for two main reasons. The Caputo derivative allows us to use the usual initial data (like $p(x, 0)=f(x)$ ), whereas the RiemannLiouville derivative requires the prior knowledge of $D^{\alpha-1} p(x, 0)=g(x)$ when the order of the equation is $\alpha$ (say $0<\alpha<1$ ). In addition to that, the Caputo derivative of a constant is zero which is not the case for the Riemann-Liouville derivative.

In the present note, we consider a generalized fractional derivative which encompasses both fractional derivatives as special cases and provides a whole range of other types of fractional derivatives in between. This derivative was introduced by Hilfer in [7-9]

$$
\left(D_{0^{+}}^{\alpha, \beta} f\right)(t)=\left(I^{\beta(1-\alpha)} \frac{d}{d t} I^{(1-\alpha)(1-\beta)} f\right)(t), \quad 0<\alpha<1,0 \leq \beta \leq 1,
$$

where

$$
\left(I_{0^{+}}^{\alpha} f\right)(t)=\frac{1}{\Gamma(\alpha)} \int_{0}^{t}(t-s)^{\alpha-1} f(s) d s, \quad t>0, \alpha>0,
$$

and therefore we name it after him. This kind of fractional derivative has already proved its usefulness (see [7-9]), and we are witnessing a growing interest in it. The parameter $\beta$ when equal to zero gives the Riemann-Liouville derivative and when it takes the value one gives the Caputo derivative. For $0<\beta<1$, we obtain many fractional derivatives interpolating these two types of well-known derivatives. 
Using the Laplace-Fourier transform, we find the explicit solution for our equation under appropriate boundary conditions. We prove here that, in our case, all these derivatives when considered in diffusion equation (1) lead to similar solutions. Consequently, there is no major benefit in treating separately these derivatives unless there is a need to unify these treatments.

For more on fractional calculus and more on interesting fractional differential equations and treatments, we refer the reader to [10-15].

In the next section, we present some material needed in our arguments. In Section 3 we consider the modified diffusion equation with Neumann boundary conditions and indicate how to find an explicit solution. Section 4 contains the Dirichlet boundary conditions case. Finally, in Section 5 we treat the infinite reservoir case. Our results are illustrated by some graphs.

\section{Preliminaries}

In this section we present some definitions and results which will be needed later in our arguments (see [12-15] for more).

Definition 1 The Riemann-Liouville fractional integral of order $\alpha$ of $f$ is defined by

$$
\left(I_{0^{+}}^{\alpha} f\right)(t)=\frac{1}{\Gamma(\alpha)} \int_{0}^{t}(t-s)^{\alpha-1} f(s) d s, \quad t>0, \alpha>0
$$

when the right-hand side exists.

Definition 2 The Riemann-Liouville fractional derivative of order $\alpha$ of $f$ is defined by

$$
\left({ }^{R L} D_{0^{+}}^{\alpha} f\right)(t)=\frac{1}{\Gamma(1-\alpha)} \frac{d}{d t} \int_{0}^{t}(t-s)^{-\alpha} f(s) d s, \quad t>0,0<\alpha<1
$$

when the right-hand side exists. Note that

$$
\left({ }^{R L} D_{0^{+}}^{\alpha} f\right)(t)=\frac{d}{d t}\left(I^{1-\alpha} f\right)(t) .
$$

Definition 3 The Caputo fractional derivative of order $\alpha$ of $f$ is defined by

$$
\left({ }^{C} D_{0^{+}}^{\alpha} f\right)(t)=\frac{1}{\Gamma(1-\alpha)} \int_{0}^{t}(t-s)^{-\alpha} f^{\prime}(s) d s, \quad t>0,0<\alpha<1
$$

(the prime here is for the derivative) when the right-hand side exists. Note that

$$
\left({ }^{C} D_{0^{+}}^{\alpha} f\right)(t)=\left(I^{1-\alpha} \frac{d}{d t} f\right)(t) .
$$

The relationship between these two types of derivatives is given by the following theorem.

Theorem 1 [12] We have

$$
\left({ }^{R L} D_{0^{+}}^{\alpha} f\right)(t)=\left({ }^{C} D_{0^{+}}^{\alpha} f\right)(t)+\frac{t^{-\alpha}}{\Gamma(1-\alpha)} f\left(0^{+}\right), \quad t>0,0<\alpha<1 .
$$


Definition 4 The Hilfer fractional derivative of $f$ of order $\alpha$ and type $\beta$ is defined by

$$
\left(D_{0^{+}}^{\alpha, \beta} f\right)(t)=\left(I^{\beta(1-\alpha)} \frac{d}{d t} I^{(1-\alpha)(1-\beta)} f\right)(t), \quad 0<\alpha<1,0 \leq \beta \leq 1
$$

whenever the right-hand side exists.

Note that when $\beta=0$,

$$
\left(D_{0^{+}}^{\alpha, 0} f\right)(t)=\frac{d}{d t}\left(I^{1-\alpha} f\right)(t)
$$

which is the Riemann-Liouville fractional derivative (see Definition 2), and when $\beta=1$,

$$
\left(D_{0^{+}}^{\alpha, 1} f\right)(t)=\left(I^{1-\alpha} \frac{d}{d t} f\right)(t)
$$

which is the Caputo fractional derivative (see Definition 3).

For $0<\alpha<1$, the Laplace transforms of these derivatives are given by

$$
\begin{aligned}
& \mathcal{L}\left[\left({ }^{R L} D_{0^{+}}^{\alpha} f\right)(t)\right](s)=s^{\alpha} \mathcal{L}[f(t)](s)-\left(I_{0^{+}}^{1-\alpha} f\right)\left(0^{+}\right), \\
& \mathcal{L}\left[\left({ }^{C} D_{0^{+}}^{\alpha} f\right)(t)\right](s)=s^{\alpha} \mathcal{L}[f(t)](s)-s^{\alpha-1} f\left(0^{+}\right), \\
& \mathcal{L}\left[\left(D_{0^{+}}^{\alpha, \beta} f\right)(t)\right](s)=s^{\alpha} \mathcal{L}[f(t)](s)-s^{\beta(\alpha-1)}\left(I_{0^{+}}^{(1-\alpha)(1-\beta)} f\right)\left(0^{+}\right), \quad 0 \leq \beta \leq 1 .
\end{aligned}
$$

It is clear that the differences in these Laplace transforms are in the 'initial' data $f\left(0^{+}\right)$, $\left(I_{0^{+}}^{1-\alpha} f\right)\left(0^{+}\right)$and $\left(I_{0^{+}}^{(1-\alpha)(1-\beta)} f\right)\left(0^{+}\right)$(this last one is natural initial data for the Hilfer derivative).

The natural space for the Hilfer fractional derivative is

$$
C_{1-\gamma}^{\alpha, \beta}[a, b]=\left\{f \in C_{1-\gamma}[a, b], D_{a^{+}}^{\alpha, \beta} f \in C_{1-\gamma}[a, b]\right\},
$$

where

$$
C_{1-\gamma}(a, b]=\left\{g:(a, b] \rightarrow \mathbf{R}:(t-a)^{1-\gamma} g(t) \in C[a, b]\right\} .
$$

However, here, since our problem is of order one, solutions must be much smoother.

\section{The problem}

We shall investigate the following linear generalized fractional diffusion problem:

$$
\left\{\begin{array}{l}
\frac{\partial p}{\partial t}(x, t)=\frac{k}{\mu \phi C_{t}} \frac{\partial^{2}}{\partial x^{2}}\left(D_{0^{+}}^{\alpha, \beta} p(x, t)\right), \quad 0 \leq x \leq L, t>0, \\
p(x, 0)=p_{i}, \quad 0 \leq x \leq L, \\
\mathfrak{q}_{0}=-\left[\frac{k A}{\mu} \frac{\partial}{\partial x}\left(D_{0^{+}}^{\alpha, \beta}\right)\right]_{x=0^{\prime}}, \quad t>0, \\
\mathfrak{q}_{L}=-\left[\frac{k A}{\mu} \frac{\partial}{\partial x}\left(D_{0^{+}}^{\alpha, \beta} p\right]_{x=L}, \quad t>0 .\right.
\end{array}\right.
$$

The different parameters $\mu, \phi, C_{t}, k$ and $A$ are positive constants which account for the viscosity, porosity, total compressibility, permeability and the area, respectively. This model describes the flow of oil in a finite reservoir. A closely related and interesting work is in [16] but for the normal form of the diffusion. 
This problem, for the Caputo fractional derivative, was derived and studied in [17]. Here we consider the more general (Hilfer) fractional derivative $D_{0^{+}}^{\alpha, \beta}$ which covers both the Riemann-Liouville derivative and the Caputo derivative (in addition to many others for $\beta$ in the interval $(0,1))$. Following the argument in [17], we introduce the dimensionless variables: $P_{D}=\frac{p_{i}-p}{p_{i}}, t_{D}=\frac{\eta t}{L^{2}}$ and $X_{D}=\frac{x}{L}$, where $\eta=\frac{k}{\mu \phi C_{t}}$.

The next two lemmas will, in particular, show that we obtain the same explicit solution using the Laplace transform as in the case of Caputo derivative (see [17]).

Lemma 1 If $t=C t_{D}$, then $D_{t}^{\mu, v} p(x, t)=C^{-\mu} D_{t_{D}}^{\mu, v} p(x, t), 0<\mu<1,0 \leq v \leq 1,0 \leq x \leq L, t>0$.

Proof From Definition 4, we have, for $0<\mu<1,0 \leq v \leq 1$,

$$
\begin{array}{rl}
D_{t}^{\mu, v} & p(x, t) \\
& =I^{v(1-\mu)} D I^{(1-\mu)(1-v)} p(x, t) \\
& =\int_{0}^{t} \frac{(t-\tau)^{v(1-\mu)-1}}{\Gamma[v(1-\mu)]} \frac{\partial}{\partial \tau}\left[\frac{1}{\Gamma[(1-\mu)(1-v)]} \int_{0}^{\tau}(\tau-s)^{(1-\mu)(1-v)-1} p(x, s) d s\right] d \tau .
\end{array}
$$

Let $t=C t_{D}$, we see that

$$
D_{t}^{\mu, v} p(x, t)=A \int_{0}^{C t_{D}}\left(C t_{D}-t\right)^{\nu(1-\mu)-1} \frac{\partial}{\partial \tau}\left[\int_{0}^{\tau}(\tau-s)^{(1-\mu)(1-\nu)-1} p(x, s) d s\right] d \tau \text {. }
$$

Put $\tau=C \xi$, then clearly

$$
\begin{aligned}
& D_{t}^{\mu, v} p(x, t) \\
& \quad=A C^{v(1-\mu)-1} \int_{0}^{t_{D}}\left(t_{D}-\xi\right)^{v(1-\mu)-1} \frac{\partial}{\partial(C \xi)}\left[\int_{0}^{C \xi}(C \xi-s)^{(1-\mu)(1-v)-1} p(x, s) d s\right] C d \xi \\
& =A C^{v(1-\mu)-1} \int_{0}^{t_{D}}\left(t_{D}-\xi\right)^{v(1-\mu)-1} \frac{\partial}{\partial \xi}\left[\int_{0}^{C \xi}(C \xi-s)^{(1-\mu)(1-v)-1} p(x, s) d s\right] d \xi .
\end{aligned}
$$

Put also $s=C u$, it appears that

$$
\begin{aligned}
D_{t}^{\mu, v} p(x, t)= & A C^{\nu(1-\mu)-1} C^{(1-\mu)(1-v)-1} \\
& \times \int_{0}^{t_{D}}\left(t_{D}-\xi\right)^{\nu(1-\mu)-1} \frac{\partial}{\partial \xi}\left[\int_{0}^{\xi}(\xi-u)^{(1-\mu)(1-v)-1} p(x, C u) C d u\right] d \xi \\
= & C^{-\mu} A \int_{0}^{t_{D}}\left(t_{D}-\xi\right)^{v(1-\mu)-1} \frac{\partial}{\partial \xi}\left[\int_{0}^{\xi}(\xi-u)^{(1-\mu)(1-v)-1} \tilde{p}(x, u) d u\right] d \xi \\
= & C^{-\mu} I^{v(1-\mu)} D I^{(1-\mu)(1-v)} \tilde{p}\left(x, t_{D}\right)=C^{-\mu} D_{t_{D}}^{\mu, \nu} p(x, t) .
\end{aligned}
$$

The proof is complete.

Lemma 2 Assume that $f(t)$ is continuous on $[0, A]$ for some $A>0$, then

$$
\lim _{t \rightarrow 0^{+}} I_{0^{+}}^{\alpha} f(t)=0, \quad \alpha>0 .
$$


Proof Let $0 \leq t \leq A$, then

$$
\begin{aligned}
\left|\int_{0}^{t}(t-s)^{\alpha-1} f(s) d s\right| & \leq \int_{0}^{t}(t-s)^{\alpha-1}|f(s)| d s \\
& \leq M \int_{0}^{t}(t-s)^{\alpha-1} d s \leq\left. M \frac{-(t-s)^{\alpha}}{\alpha}\right|_{0} ^{t}=\frac{M}{\alpha} t^{\alpha},
\end{aligned}
$$

where $M$ is a bound for $f(t)$ on $[0, A]$.

In view of our problem (5), the solution $p(x, t)$ must be at least absolutely continuous. Its continuity nearby zero implies by Lemma 2 that $I_{0+}^{1-\alpha} f\left(0^{+}\right)=I_{0+}^{(1-\alpha)(1-\beta)} f\left(0^{+}\right)=0$. Therefore all three derivatives have equal Laplace transforms (see (2)-(4)) (if in addition $f\left(0^{+}\right)=0$ ). This fact together with Lemma 1 shows that the argument in [17] is valid without any changes.

Observe that the change of variables has lead to $p\left(x_{D}, 0\right)=0$, which by Theorem $1 \mathrm{implies}$ that both the Riemann-Liouville derivative and the Caputo derivative are equal. Another way to see this fact is from the next two lemmas.

Definition 5 A function $f(t) \in L^{1}(a, b)$ (the space of summable functions) is said to have a summable fractional derivative $D_{0^{+}}^{\alpha} f$ if $I_{0+}^{1-\alpha} f \in A C([a, b])$ (the space of absolutely continuous functions).

Lemma 3 [18, p.48] If $f(t)$ has a summable fractional derivative ${ }^{R L} D_{0^{+}}^{\beta} f$ with $0<\beta<1$, then we have

$$
I_{0+}^{\alpha} R L D_{0^{+}}^{\beta} f(t)=I_{0+}^{\alpha-\beta} f(t)-\frac{I^{1-\beta} f\left(0^{+}\right)}{\Gamma(\alpha)} t^{\alpha-1} .
$$

Lemma 4 [12, p.95] If $f(t) \in C[a, b], 0<\alpha<1$, then $\left({ }^{C} D_{0^{+}}^{\alpha}{ }^{\alpha} f\right)(t)=f(t), t \in[a, b]$.

Now, Definition 3, Definition 4 and Lemma 3 imply that

$$
\begin{aligned}
D^{\alpha, \beta} f(t) & =I^{\beta(1-\alpha)} D I^{(1-\alpha)(1-\beta)} f(t)={ }^{C} D^{1-\beta(1-\alpha)} I^{(1-\alpha)(1-\beta)} f(t) \\
& ={ }^{C} D^{1-\beta(1-\alpha)} I^{1-\beta(1-\alpha)-\alpha} f(t) \\
& ={ }^{C} D^{1-\beta(1-\alpha)}\left[I^{1-\beta(1-\alpha) R L} D^{\alpha} f(t)+\frac{I^{1-\alpha} f\left(0^{+}\right)}{\Gamma(1-\beta(1-\alpha))} t^{-\beta(1-\alpha)}\right] .
\end{aligned}
$$

This holds if $f(t)$ has a summable fractional derivative $D_{0^{+}}^{\alpha} f(t)$ that is $I_{0^{+}}^{1-\alpha} f \in A C([0, T])$, which is the case when $f$ itself is absolutely continuous on $[0, T]$ for some $T>0$. In our case, $p(x, t)$ is absolutely continuous. Lemma 2 ensures that $I_{0^{+}}^{1-\alpha} f(0)=0$. Therefore,

$$
D_{0^{+}}^{\alpha, \beta} f(t)={ }^{C} D^{1-\beta(1-\alpha)} I^{1-\beta(1-\alpha) R L} D_{0+}^{\alpha} f(t)={ }^{R L} D_{0+}^{\alpha} f(t)
$$

by Lemma 4 if ${ }^{R L} D_{0^{\alpha}}^{\alpha} f(t)$ is continuous. This is the case if $f\left(0^{+}\right)=0$ and $f$ is continuously differentiable because $I_{0^{+}}^{1-\alpha}$ maps $C[0, T]$ into $C[0, T]$ (it also maps $C_{\gamma}[0, T]$ into $C[0, T]$, see [19]). 


\section{Dirichlet boundary conditions}

Consider the problem

$$
\left\{\begin{array}{l}
\frac{\partial p}{\partial t}=k \frac{\partial^{2}}{\partial x^{2}}\left(D^{\alpha, \beta} p\right)+f(x, t), \quad 0 \leq x \leq L, t>0 \\
p(x, 0)=p_{i}, \quad 0 \leq x \leq L \\
p(0, t)=g(t), \quad t>0 \\
p(L, t)=h(t), \quad t>0
\end{array}\right.
$$

where $g(t), h(t)$ are continuously differentiable and $f(x, t)$ is a forcing term.

Putting $p(x, t)=P(x, t)+u(x, t)$, with

$$
u(x, t)=g(t)+\frac{x}{L}[h(t)-g(t)]
$$

we see that

$$
\frac{\partial}{\partial t}[P(x, t)+u(x, t)]=k \frac{\partial^{2}}{\partial x^{2}}\left(D^{\alpha, \beta} P(x, t)+D^{\alpha, \beta} u(x, t)\right)+f(x, t)
$$

implies

$$
\frac{\partial P}{\partial t}(x, t)=k \frac{\partial^{2}}{\partial x^{2}}\left(D^{\alpha, \beta} P(x, t)\right)+k D^{\alpha, \beta} \frac{\partial^{2} u}{\partial x^{2}}-\frac{\partial u}{\partial t}+f(x, t) .
$$

Clearly,

$$
\frac{\partial^{2} u}{\partial x^{2}}=0 \quad \text { and } \quad \frac{\partial u}{\partial t}=g^{\prime}(t)+\frac{x}{L}\left[h^{\prime}(t)-g^{\prime}(t)\right] .
$$

Moreover,

$$
p_{i}=p(x, 0)=P(x, 0)+u(x, 0)=P(x, 0)+g(0)+\frac{x}{L}[h(0)-g(0)] .
$$

So

$$
P(x, 0)=p_{i}-u(x, 0)=p_{i}-g(0)-\frac{x}{L}[h(0)-g(0)] .
$$

In addition to that, we have

$$
P(0, t)=P(L, t)=0 .
$$

Therefore from (8)-(11), $P(x, t)$ satisfies

$$
\left\{\begin{array}{l}
\frac{\partial P}{\partial t}=k \frac{\partial^{2}}{\partial x^{2}}\left(D^{\alpha, \beta} P\right)+f^{*}(x, t), \quad 0 \leq x \leq L, t>0 \\
P(x, 0)=P^{*}(x), \quad 0 \leq x \leq L \\
P(0, t)=P(L, t)=0, \quad t>0
\end{array}\right.
$$

where

$$
f^{*}(x, t)=f(x, t)-\frac{\partial u}{\partial t}=f(x, t)-g^{\prime}(t)-\frac{x}{L}\left[h^{\prime}(t)-g^{\prime}(t)\right]
$$


and

$$
P^{*}(x)=p_{i}-g(0)-\frac{x}{L}[h(0)-g(0)] .
$$

Definition 6 The finite Fourier sine transform of $f(x), 0<x<L$, is defined by

$$
F_{s}(n)=\int_{0}^{L} f(x) \sin \frac{n \pi x}{L} d x
$$

and its inverse is

$$
f(x)=\frac{2}{L} \sum_{n=1}^{\infty} F_{s}(n) \sin \frac{n \pi x}{L}
$$

Theorem 2 The following holds:

$$
F_{s}\left(\frac{\partial v}{\partial x}\right)=-\frac{n \pi}{L} F_{c}(v)
$$

where $F_{c}$ is the finite Fourier cosine transform and

$$
F_{s}\left(\frac{\partial^{2} v}{\partial x^{2}}\right)=-\frac{n \pi}{L} F_{c}\left(\frac{\partial v}{\partial x}\right)=-\frac{n^{2} \pi^{2}}{L^{2}} F_{s}(v)+\frac{n \pi}{L}[v(0, t)-v(L, t) \cdot \cos n \pi] .
$$

Applying first the Laplace transform to the equation in (12), we find

$$
s \bar{P}(x, s)-P(x, 0)=k \frac{\partial^{2}}{\partial x^{2}}\left[s^{\alpha} \bar{P}(x, s)-s^{\beta(\alpha-1)}\left(I^{(1-\alpha)(1-\beta)} \bar{P}\right)\left(0^{+}\right)\right]+\bar{f}^{*}(x, s),
$$

where $\bar{P}$ and $\bar{f}^{*}$ denote the Laplace transforms of $P$ and $f^{*}$, respectively.

In view of Lemma 2, equation (13) reduces to

$$
s \bar{P}(x, s)=k s^{\alpha} \frac{\partial^{2} \bar{P}}{\partial x^{2}}(x, s)+\bar{f}^{*}(x, s)+P^{*}(x) .
$$

Next, we apply the Fourier sine transform to (14) to get

$$
s \widehat{\bar{P}}(n, s)=-\frac{n^{2} \pi^{2} k}{L^{2}} s^{\alpha} \widehat{\bar{P}}(n, s)+F_{s}\left[\bar{f}^{*}(x, s)+P^{*}(x)\right]
$$

where the 'hat' is for the Fourier sine transform. Therefore,

$$
\widehat{\bar{P}}(n, s)=\frac{s^{-\alpha} F_{s}\left[\bar{f}^{*}(x, s)+P^{*}(x)\right]}{s^{1-\alpha}+\frac{n^{2} \pi^{2} k}{L^{2}}} .
$$

From the relation

$$
F_{s}(a+b x)=\frac{L}{n \pi}\left[a-(-1)^{n}(a+b L)\right]
$$


it follows that

$$
F_{s}\left(P^{*}(x)\right)=\frac{L}{n \pi}\left[p_{i}-g(0)-(-1)^{n}\left(p_{i}-h(0)\right)\right] .
$$

Furthermore, it is easy to see that

$$
\begin{aligned}
F_{s}\left[\bar{f}^{*}(x, s)\right] & =F_{s}\left[\bar{f}(x, s)-\bar{g}^{\prime}(s)-\frac{x}{L}\left(\bar{h}^{\prime}(s)-\bar{g}^{\prime}(s)\right)\right] \\
& =\hat{\bar{f}}(n, s)-F_{s}\left[\bar{g}^{\prime}(s)+\frac{x}{L}\left(\bar{h}^{\prime}(s)-\bar{g}^{\prime}(s)\right)\right] \\
& =\hat{\bar{f}}(n, s)-\frac{L}{n \pi}\left[\bar{g}^{\prime}(s)-(-1)^{n} \bar{h}^{\prime}(s)\right] .
\end{aligned}
$$

Using the inverse of the Fourier sine transform, we find

$$
\bar{P}(x, s)=\frac{2}{L} \sum_{n=1}^{\infty} \widehat{\bar{P}}(n, s) \sin \frac{n \pi x}{L},
$$

and then, inverting the Laplace transform, we obtain

$$
P(x, t)=\frac{2}{L} \sum_{n=1}^{\infty} \mathcal{L}^{-1}\left(\widehat{\bar{P}}(n, s) \sin \frac{n \pi x}{L}\right) .
$$

In view of (15) we have

$$
\begin{aligned}
\mathcal{L}^{-1}[\widehat{\bar{P}}(n, s)]= & \mathcal{L}^{-1}\left[\frac{s^{-\alpha} F_{s}\left[\bar{f}^{*}(x, s)\right]}{s^{1-\alpha}+\frac{n^{2} \pi^{2} k}{L^{2}}}\right]+\mathcal{L}^{-1}\left[\frac{s^{-\alpha} F_{s}\left[P^{*}(x)\right]}{s^{1-\alpha}+\frac{n^{2} \pi^{2} k}{L^{2}}}\right] \\
= & \mathcal{L}^{-1}\left[\frac{s^{-\alpha} \hat{\bar{f}}(n, s)}{s^{1-\alpha}+\frac{n^{2} \pi^{2} k}{L^{2}}}\right]-\mathcal{L}^{-1}\left[\frac{L s^{-\alpha}}{n \pi} \frac{\left(\bar{g}^{\prime}(s)-(-1)^{n} \bar{h}^{\prime}(s)\right)}{s^{1-\alpha}+\frac{n^{2} \pi^{2} K}{L^{2}}}\right] \\
& +\mathcal{L}^{-1}\left[\frac{L}{n \pi}\left(p_{i}-g(0)-(-1)^{n}\left(p_{i}-h(0)\right)\right) \frac{s^{-\alpha}}{s^{1-\alpha}+\frac{n^{2} \pi^{2} k}{L^{2}}}\right] .
\end{aligned}
$$

Now, appealing to the formula

$$
\mathcal{L}\left[t^{\beta-1} E_{\alpha, \beta}\left( \pm a t^{\alpha}\right)\right]=\frac{s^{\alpha-\beta}}{s^{\alpha} \mp a}
$$

we see that

$$
\mathcal{L}^{-1}\left[\frac{s^{-\alpha}}{s^{1-\alpha}+\frac{n^{2} \pi^{2} k}{L^{2}}}\right]=E_{1-\alpha, 1}\left(-\frac{n^{2} \pi^{2} k}{L^{2}} t^{1-\alpha}\right)
$$

Further

$$
\mathcal{L}^{-1}\left[\frac{s^{-\alpha} \hat{\bar{f}}(n, s)}{s^{1-\alpha}+\frac{n^{2} \pi^{2} k}{L^{2}}}\right]=\int_{0}^{t} \hat{f}(n, t-s) E_{1-\alpha, 1}\left(-\frac{n^{2} \pi^{2} k}{L^{2}} s^{1-\alpha}\right) d s
$$


and

$$
\begin{aligned}
\mathcal{L}^{-1} & {\left[\frac{s^{-\alpha}\left(\bar{g}^{\prime}(s)-(-1)^{n} \bar{h}^{\prime}(s)\right)}{s^{1-\alpha}+\frac{n^{2} \pi^{2} k}{L^{2}}}\right] } \\
& =\int_{0}^{t}\left[g^{\prime}(t-s)-(-1)^{n} h^{\prime}(t-s)\right] E_{1-\alpha, 1}\left(-\frac{n^{2} \pi^{2} k}{L^{2}} s^{1-\alpha}\right) d s .
\end{aligned}
$$

Taking into account (20)-(22) in (19), we obtain

$$
\begin{aligned}
\mathcal{L}^{-1}[\widehat{\bar{P}}(n, s)]= & \int_{0}^{t}\left\{\hat{f}(n, t-s)-\left[g^{\prime}(t-s)-(-1)^{n} h^{\prime}(t-s)\right]\right\} E_{1-\alpha, 1}\left(-\frac{n^{2} \pi^{2} k}{L^{2}} s^{1-\alpha}\right) d s \\
& +\frac{L}{n \pi}\left[p_{i}-g(0)-(-1)^{n}\left(p_{i}-h(0)\right)\right] E_{1-\alpha, 1}\left(-\frac{n^{2} \pi^{2} k}{L^{2}} t^{1-\alpha}\right) .
\end{aligned}
$$

Finally, relation (18) leads to

$$
\begin{aligned}
P(x, t)= & \frac{2}{L} \sum_{n=1}^{\infty} \int_{0}^{t}\left\{\hat{f}(n, t-s)-\left[g^{\prime}(t-s)-(-1)^{n} h^{\prime}(t-s)\right]\right\} \\
& \times E_{1-\alpha, 1}\left(-\frac{n^{2} \pi^{2} k}{L^{2}} s^{1-\alpha}\right) d s \sin \frac{n \pi x}{L} \\
& +\frac{2}{L} \sum_{n=1}^{\infty} \frac{L}{n \pi}\left[p_{i}-g(0)-(-1)^{n}\left(p_{i}-h(0)\right)\right] \\
& \times E_{1-\alpha, 1}\left(-\frac{n^{2} \pi^{2} k}{L^{2}} t^{1-\alpha}\right) \sin \frac{n \pi x}{L}
\end{aligned}
$$

and $p(x, t)=P(x, t)+u(x, t)$ gives the explicit solution of problem (7).

\section{Explicit solution in an infinite reservoir}

In this section we consider a similar problem but in an infinite reservoir, which is the case in many practical situations.

$$
\left\{\begin{array}{l}
\frac{\partial p}{\partial t}(x, t)=k \frac{\partial^{2}}{\partial x^{2}}\left(D^{\alpha, \beta} p(x, t)\right)+f(x, t), \quad-\infty<x<\infty, t>0 \\
p(x, 0)=g(x), \quad-\infty<x<\infty \\
p( \pm \infty, t)=0, \quad t>0
\end{array}\right.
$$

Figure 1 The graph of $P(x, t)$ when $\alpha=0.2$.

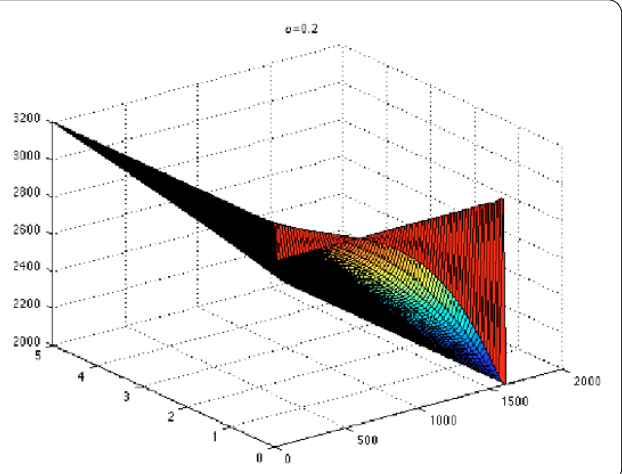


Figure 2 The graph of $P(x, t)$ when $\alpha=0.5$.

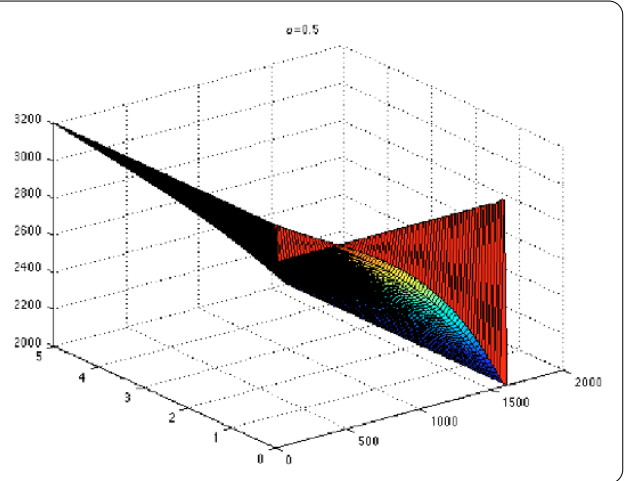

Figure 3 The graph of $P(x, t)$ when $\alpha=0.8$.

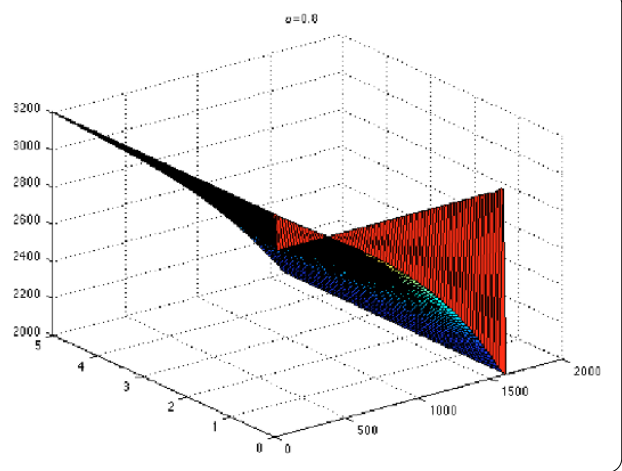

Figure 4 The graph of $P(x, t)$ when $\alpha=0.95$.

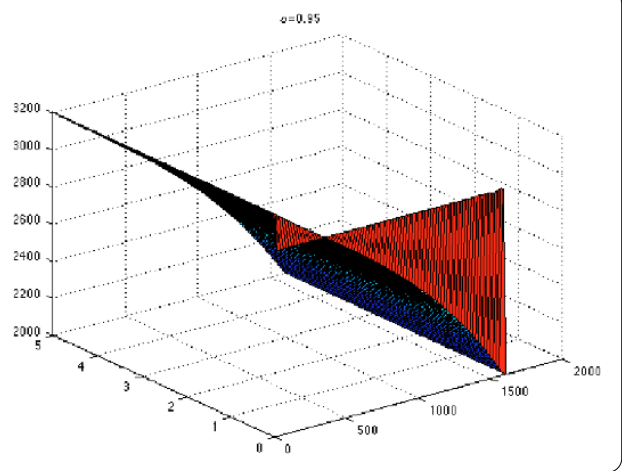

The Laplace transform with respect to the time variable applied to the equation in (25) gives

$$
s \bar{p}(x, s)-p(x, 0)=k \frac{\partial^{2}}{\partial x^{2}}\left[s^{\alpha} \bar{p}(x, s)-s^{\beta(\alpha-1)}\left(I^{(1-\alpha)(1-\beta)} p\right)\left(x, 0^{+}\right)\right]+\bar{f}(x, s)
$$

As $p(x, 0)=g(x)$ and $\left(I^{(1-\alpha)(1-\beta)} p\right)(x, 0)=0$, by the continuity of $p(x, t)$ nearby $t=0$ (see Lemma 2), we get

$$
s \bar{p}(x, s)=k s^{\alpha} \frac{\partial^{2} \bar{p}}{\partial x^{2}}(x, s)+\bar{f}(x, s)+g(x)
$$



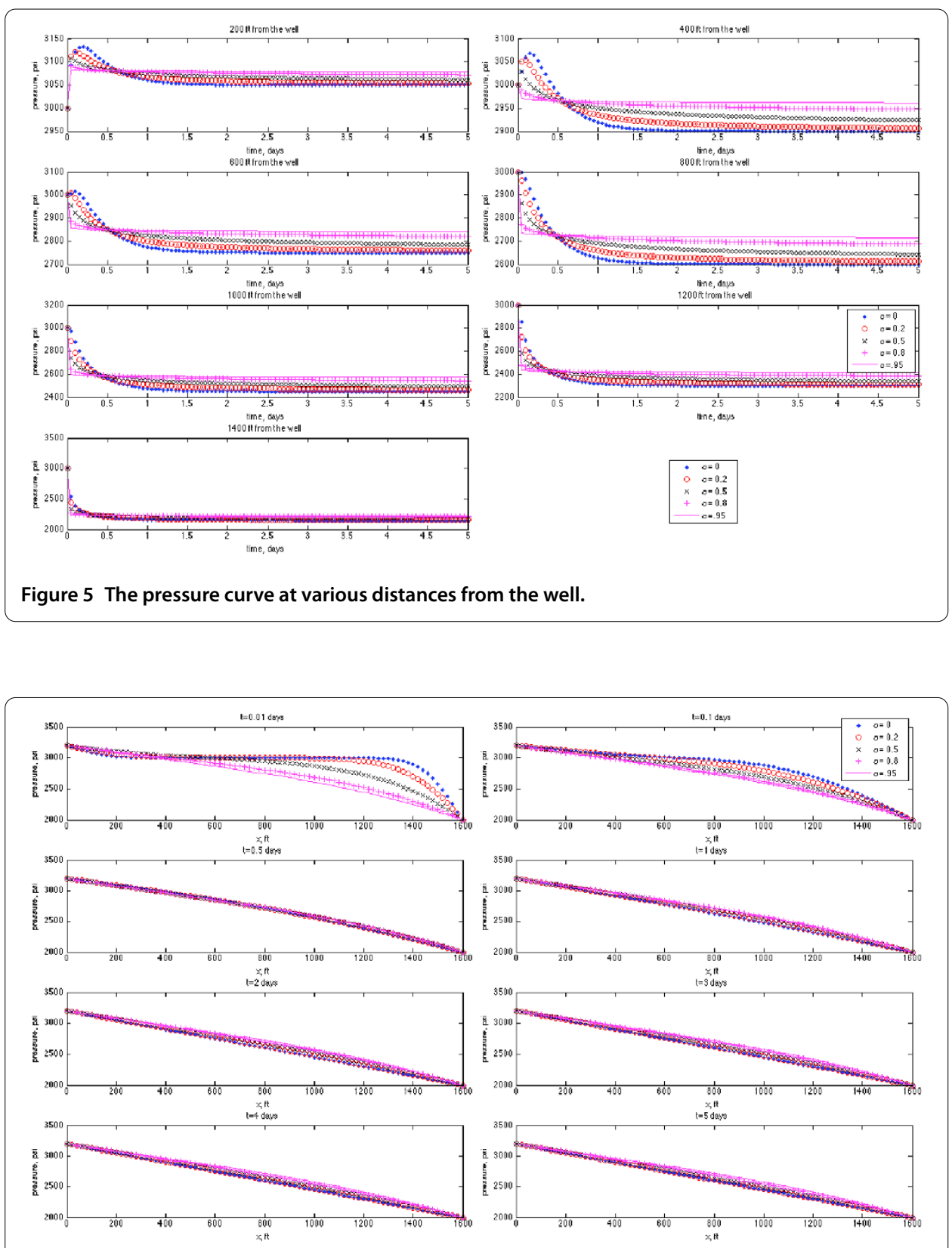

Figure 6 The pressure curve at various times.

Next, we apply the Fourier transform $\left(\hat{f}(y)=\frac{1}{\sqrt{2 \pi}} \int_{-\infty}^{+\infty} f(x) e^{i x y} d x\right)$ to this last equation (26) to arrive at

$$
s \hat{\bar{p}}(y, s)=-k s^{\alpha} y^{2} \hat{\bar{p}}(y, s)+\hat{\bar{f}}(y, s)+\hat{g}(y) .
$$

Therefore

$$
\left[s+k s^{\alpha} y^{2}\right] \hat{\bar{p}}(y, s)=\hat{\bar{f}}(y, s)+\hat{g}(y)
$$


and hence

$$
\hat{\bar{p}}(y, s)=\frac{\hat{\bar{f}}(y, s)}{s+k s^{\alpha} y^{2}}+\frac{\hat{g}(y)}{s+k s^{\alpha} y^{2}} .
$$

Now, the inverse Laplace transform of (27) yields

$$
\hat{p}(y, t)=\int_{0}^{t} E_{1-\alpha, 1}\left(-k y^{2}(t-s)^{1-\alpha}\right) \hat{f}(y, s) d s+\hat{g}(y) E_{1-\alpha, 1}\left(-k y^{2} t^{1-\alpha}\right),
$$

and the inverse Fourier transform gives

$$
\begin{aligned}
p(x, t)= & \int_{-\infty}^{\infty}\left(\int_{0}^{t} E_{1-\alpha, 1}\left(-k y^{2}(t-s)^{1-\alpha}\right) \hat{f}(y, s) d s\right) \cdot e^{-i y x} d y \\
& +\int_{-\infty}^{\infty} \hat{g}(y) E_{1-\alpha, 1}\left(-k y^{2} t^{1-\alpha}\right) e^{-i y x} d y .
\end{aligned}
$$

Finally we mention that this type of fractional derivative was also studied in [20] and refer the reader to $[21,22]$ for other interesting problems and methods.

The graphs in Figures 1-6 correspond to different orders and the following data: $f(x, t)=$ $0, P(x, 0)=3,000, P(0, t)=3,200, P(1,600, t)=2,000, k=7.5937 \mathrm{e}+005$.

\section{Competing interests}

The authors declare that they have no competing interests.

\section{Authors' contributions}

SA and NT solved the problems. RAG participated in establishing the explicit solution and carried out the numerical treatment. All authors read and approved the final manuscript.

\section{Author details}

${ }^{1}$ Department of Mathematics and Statistics, King Fahd University of Petroleum and Minerals, Dhahran, 31261, Saudi Arabia. ${ }^{2}$ Department of Mathematics, University of Pittsburgh at Greensburg, Greensburg, PA 15601, USA

\section{Acknowledgements}

The authors are grateful for the financial support and the facilities provided by King Fahd University of Petroleum and Minerals and King Abdulaziz City for Science and Technology (KACST) NSTIP research grant No. 11-OIL1663-04.

Received: 5 June 2013 Accepted: 7 November 2013 Published: 03 Dec 2013

\section{References}

1. Darcy, H: Les Fontaines Publiques de la Ville de Dijon. Dalmont, Paris (1856)

2. King, M: Darcy's law and the field equations of the flow of underground fluids. Bull. Int. Assoc. Sci. Hydrol. 2(1), 23-59 (1957)

3. Caputo, M: Diffusion of fluids in porous media with memory. Geothermics 28, 113-130 (1999)

4. Metzler, R, Klafter, J: The random walk's guide to anomalous diffusion: a fractional dynamics approach. Phys. Rep. 339 $1-77(2000)$

5. Salim, TO, El-Kahlout, A: Solution of fractional order Rayleigh-Stokes equations. Adv. Theor. Appl. Mech. 1(5), 241-254 (2008)

6. Langlands, TAM: Solution of a modified fractional diffusion equation. Physica A 367, 136-144 (2006)

7. Hilfer, R: Fractional time evolution. In: Hilfer, R (ed.) Applications of Fractional Calculus in Physics, p. 87. World Scientific, Singapore (2000)

8. Hilfer, R: Fractional calculus and regular variation in thermodynamics. In: Hilfer, R (ed.) Applications of Fractional Calculus in Physics, p. 429. World Scientific, Singapore (2000)

9. Hilfer, R: Experimental evidence for fractional time evolution in glass materials. Chem. Phys. 284, 399-408 (2002)

10. Gorenflo, R, Mainardi, F: Fractional calculus: integral and differential equations of fractional orders. In: Fractals and Fractional Calculus in Continuum Mechanics. Springer, New York (1997)

11. Herzallah, MAE, El-Sayed, AMA, Baleanu, D: On the fractional-order diffusion-wave process. Rom. J. Phys. 55(3-4), 274-284 (2010)

12. Kilbas, AA, Srivastava, HM, Trujillo, TJ: Theory and Applications of Fractional Differential Equations. North-Holland Mathematics Studies, vol. 204, van Mill, J (ed.). Elsevier, Amsterdam (2006)

13. Miller, KS, Ross, B: An Introduction to the Fractional Calculus and Fractional Differential Equations. Wiley, New York (1993) 
14. Oldham, KB, Spanier, J: The Fractional Calculus. Academic Press, New York (1974)

15. Podlubny, I: Fractional Differential Equations. Mathematics in Sciences and Engineering, vol. 198. Academic Press, San Diego (1999)

16. Sandev, T, Metzler, R, Tomovski, Z: Fractional diffusion equation with a generalized Riemann-Liouville time fractional derivative. J. Phys. A, Math. Theor. 44, 255203 (2011)

17. Malek, NA, Ghanam, RA, Al-Homidan, S: Sensitivity of the pressure distribution to the fractional order $\alpha$ in the fractional diffusion equation (submitted)

18. Samko, SG, Kilbas, AA, Marichev, Ol: Fractional Integrals and Derivatives. Theory and Applications. Gordon \& Breach, Yverdon (1993)

19. Kou, C, Liu, J, Ye, Y: Existence and uniqueness of solutions for the Cauchy-type problems of fractional differential equations. Discrete Dyn. Nat. Soc. 2010, Article ID 142175 (2010)

20. Furati, KM, Kassim, MD, Tatar, N-e: Existence and uniqueness for a problem involving Hilfer fractional derivative. Comput. Math. Appl. 64(6), 1616-1626 (2012)

21. Baleanu, D, Diethelm, K, Scalas, E, Trujillo, JJ: Fractional Calculus Models and Numerical Methods. Series on Complexity, Nonlinearity and Chaos. World Scientific, Singapore (2012)

22. Baleanu, D, Machado, JAT, Luo, AC: Fractional Dynamics and Control. Springer, Berlin (2012)

10.1186/1687-1847-2013-349

Cite this article as: Al-Homidan et al.: On a generalized diffusion equation arising in petroleum engineering. Advances in Difference Equations 2013, 2013:349

\section{Submit your manuscript to a SpringerOpen ${ }^{\circ}$ journal and benefit from:}

- Convenient online submission

Rigorous peer review

- Immediate publication on acceptance

- Open access: articles freely available online

- High visibility within the field

- Retaining the copyright to your article 